WHY UPWINDING IS REASONABLE

Myron B. Allen

Proceedings Paper

WWRC-84-17

In

FINITE ELEMENTS IN WATER RESOURCES

Proceedings of the 5 th International

Conference, Burlington, Vermont, U.S.A.

June 1984

Myron B. Allen

Department of Mathematics

University of Wyoming

Laramie, Wyoming 


\section{Finite Elements \\ in Water \\ Resources}

Proceedings of the 5th International Conference, Burlington, Vermont, U.S.A., June 1984

Editors:

J.P. Laible

C.A. Brebbia

W. Gray

G.Pinder

A Computational Mechanics Centre Publication Springer-Verlag

Berlin Heidelberg New York Tokyo 


\title{
WHY UPWINDING IS REASONABLE
}

\author{
Myron B. Allen
}

University of Wyoming

\section{INTRODUCTION}

Upwind-biased discrete approximations have a distinguished history in numerical fluid mechanics, dating at least to von Neumann and Richtmyer (1950). Lately, however, upwinding has come under fire in water resources engineering. Among the most effective critics of upwind techniques are Gresho and Lee (1980), who take umbrage at the smearing of steep gradients in solutions of partial differential equations. While this viewpoint has cogency, a blanket condemnation of upwinding would be injudicious. There exist fluid flows for which upstream-biased discretizations are not only valid but in fact mathematically more appropriate than central approximations having higher-order accuracy.

Figures 1 and 2 illustrate the source of the controversy. Both plots show numerical solutions to a convection-dominated species transport equation using finite-element collocation. Figure 1, the result of a centered scheme, shows a solution having unrealistic wiggles near the concentration front; Figure 2, from an upwind scheme, exhibits nonphysical smearing. The wiggles in the centered scheme disappear altogether when the spatial step $\Delta x$ is small enough, whereas the smearing associated with upstream weighting decreases continuously with $\Delta x$. Gresho and Lee argue that the wiggles indicate an inappropriate spatial grid and that suppressing them via upwinding eliminates useful symptom in favor of a less informative flaw, smearing.

Were wiggles the only difficulty with centered schemes, proscribing upwind methods might be in order. However, as we shall see, for certain types of equations centered schemes can fail to converge. This difficulty is not symptomatic of an unsuitable grid; rather, it betrays an inability of centered schemes to impose proper uniqueness criteria. For such equations, upwinding can be reasonable.

\section{SOME UPWINDING TECHNIQUES}

Of various discrete methods used in numerical fluid mechanics, finite differences, Galerkin methods, and finite-element collocation have proved to be among the most attractive. A brief review of techniques in each of these three categuries shows how upwirdiag generates swear ig. To 
u

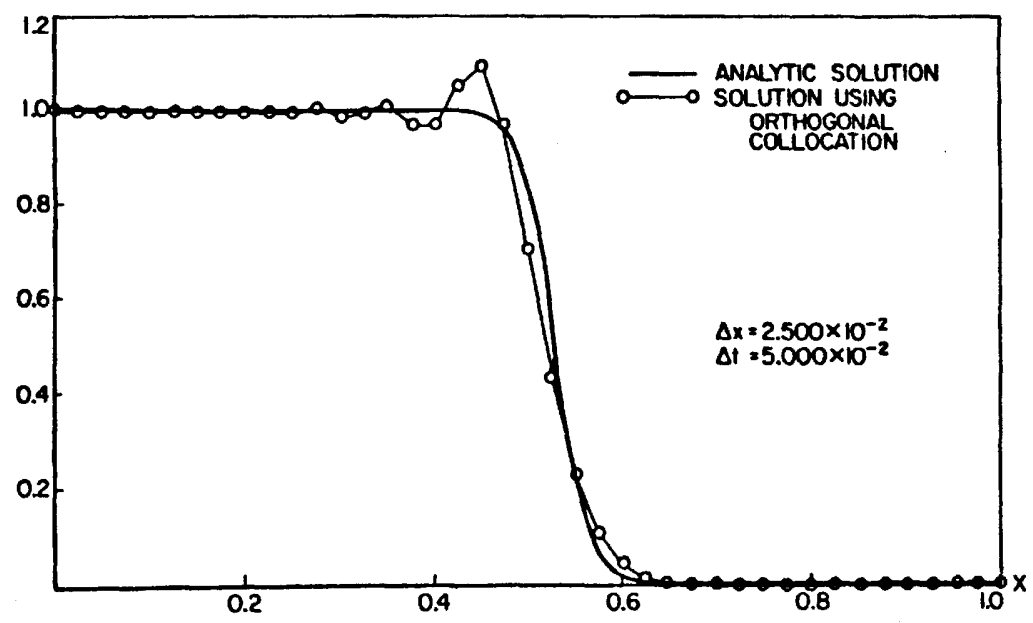

Figure 1. Solution to the convection-diffusion equation using standard collocation on finite elements.

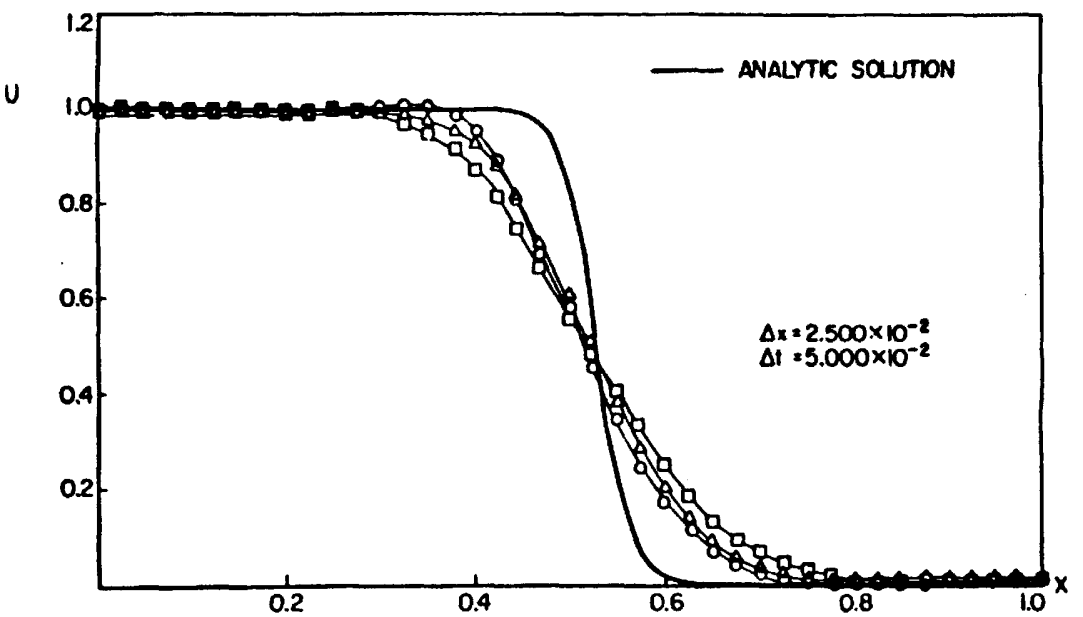

Figure 2. Solutions to the convection-diffusion equation using several choices of upstream collocation points. 
make matters concrete, consider as a paradigm of convection-dominated flows the constant-coefficient convection-diffusion problem

$$
\begin{gathered}
\partial u / \partial t+\partial u / \partial x-P e^{-1} \partial 2 u / \partial x^{2}=0 \\
u(x, 0)=0, x \in(0,1) \\
u(0, t)=1 \text { and } \partial u(1, t) / \partial x=0, t \geq 0
\end{gathered}
$$

where $x$ and $t$ are dimensionless space and time coordinates, $u(x, t)$ denotes a normalized concentration, and the Peclet number Pe measures the degree to which convection dominates diffusion.

As commonly implemented, each of the discrete interior methods calls for a partition $\Delta_{N}: 0=x_{0}<x_{1}<\ldots<x_{N+1}=1$ of the spatial domain. Suppose for simplicity that $\Delta_{N}$ has uniform mesh $\Delta x$. Then the following finitedifference analog of Equation (1) has truncation error $O\left(\Delta x^{2}\right)$ :

$$
d u_{i} / d t=-(2 \Delta x)^{-1}\left(u_{i+1}-u_{i-1}\right)+\left(\operatorname{Pe} \Delta x^{2}\right)^{-1}\left(u_{i+1}-2 u_{i}+u_{i-1}\right)
$$

where $u_{i}(t)$ signifies the approximate solution at $x=i \Delta x$ and time $t$. The problem with Equation (2) is that, unless $\Delta \mathbf{x}$ is sufficiently small, the numerical solution exhibits spurious wiggles near sharp concentration fronts. A desire to avoid these wiggles in favor of smearing prompts many analysts to resort to upwind schemes.

The simplest way to derive an upwind scheme from Equation (2) is to replace the analog of the convective term $\partial u / \partial x$ by a one-sided difference, yielding

$$
d u_{i} / d t=-\Delta x^{-1}\left(u_{i}-u_{i-1}\right)+\left(P e \Delta x^{2}\right)^{-1}\left(u_{i+1}-2 u_{i}+u_{i-1}\right)
$$

The truncation error of Equation (3), $O(\Delta x)$, is larger than that for Equation (2), and writing the lowest error term explicitly shows

$$
\begin{aligned}
d u_{i} / d t & +\Delta x^{-1}\left(u_{i}-u_{i}-1\right)-\left(P e \Delta x^{2}\right)^{-1}\left(u_{i+1}-2 u_{i}+u_{j-1}\right) \\
= & \partial u / \partial t+\partial u / \partial x-\left(P e^{-1}+\Delta x / 2\right) \partial^{2} u / \partial x^{2}+O\left(\Delta x^{2}\right)
\end{aligned}
$$

where the terms on the right are evaluated at $x=x_{i}$. It is clear that the upwind difference scheme augments physical diffusion by an amount proportional to $\Delta x$. Hence by sacrificing one order of spatial accuracy one can suppress nonphysical wiggles at the cost of numerically induced dissipation.

Upwinding techniques also exist for finite-element Galerkin schemes. One such technique is the use of upstream-biased test functions proposed by Heinrich et al. (1977). Consider the standard Calerkin method applied to Equation (1). This method seeks a trial function

$$
\hat{u}(x, t)=u_{\partial}(x)+\sum_{i=1}^{N} u_{i}(t) L_{i}(x)
$$

approximating the true solution $u(x, t)$. Here $u_{\partial}(x)$ is a chapeau function on $\Delta N$ satisfying the boundary conditions and vanishing at each interior node $x_{i}$, $i=1, \ldots, N$, and the functions $L_{i}(x)$ are elements of the chapeau basis on $\Delta N$. 
To determine the nodal values $u_{i}(t)$, we force the residual $\partial \hat{u} / \partial t+\partial \hat{u} / \partial x$ - $P e^{-1} \partial^{2} \hat{u} / \partial x^{2}$ to be orthogonal to each basis function $L_{j}(x), j=1, \ldots, N$, with respect to the inner product $\langle f, g\rangle=\int_{0}^{1} f(x) g(x) d x$. This requirement leads to a set of $N$ ordinary differential equations for the $u_{i}(t)$ :

$$
\sum_{i=1}^{N}\left(\left\langle L_{i}, L_{j}\right\rangle d u_{i} / d t+\left\langle L_{i}{ }^{\prime}, L_{j}\right\rangle u_{i}+P e^{-1}\left\langle L_{i}{ }^{\prime}, L_{j}{ }^{\prime}\right\rangle u_{i}\right)=0, j=1, \ldots, N
$$

Computing the integrals then gives

$$
\begin{gathered}
\frac{\Delta x}{6} \frac{d}{d t}\left(u_{j-1}+4 u_{j}+u_{j+1}\right)+\frac{1}{2}\left(u_{j+1}-u_{j-1}\right) \\
-(\Delta x P e)^{-1}\left(u_{j+1}-2 u_{j}+u_{j-1}\right)=0
\end{gathered}
$$

Equation (6) is similar to the centered difference formula, Equation (3), the only difference being a peculiar spatial average of time derivatives in Equation (6). This scheme, like Equation (3), produces wiggles when $\Delta x$ is too large.

The method advanced by Heinrich et al. modifies Equation (5) by replacing the test functions in the convective term with asymmetric functions $L_{j}{ }^{*}(x)=$ $L_{j}(x)+\alpha A_{j}(x)$, where

$$
A_{j}(x)=\left\{\begin{array}{l}
2\left[\Delta x^{-2}\left(x-x_{j-1}\right)^{2}-\Delta x^{-1}\left(x-x_{j-1}\right)\right], x \in\left[x_{j-1}, x_{j}\right] \\
-2\left[\Delta x^{-2}\left(x-x_{j}\right)^{2}-\Delta x^{-1}\left(x-x_{j}\right)\right], x \in\left[x_{j}, x_{j}+1\right] \\
0, x \notin\left[x_{j-1}, x_{j+1}\right]
\end{array}\right.
$$

and $\alpha\rangle 0$. Thus the integral $\left\langle L_{i}{ }^{\prime}, L_{i}{ }^{*}\right\rangle$ appears in Equation (5) instead of $\left\langle L_{i}, L_{j}\right\rangle$, and the upstream-weighted Calerkin scheme differs from Equation (6) by $\alpha\left\langle L_{i}{ }^{\prime}, A_{j}\right\rangle$. Simple calculation shows

$$
\left\langle L_{i}, A_{j}\right\rangle=\left\{\begin{array}{l}
-1 / 2, i=j \pm 1 \\
1, i=j \\
0,|i-j|>1
\end{array}\right.
$$

so the scheme proposed by Heinrich et al. reduces to

$$
\begin{aligned}
& \frac{\Delta x}{6} \frac{d}{d t}\left(u_{j-1}+4 u_{j}+u_{j+1}\right)+\frac{1}{2}\left(u_{j+1}-u_{j-1}\right) \\
& -\Delta x^{-1}\left(P e^{-1}+\alpha \Delta x / 2\right)\left(u_{j+1}-2 u_{j}+u_{j-1}\right)
\end{aligned}
$$

Therefore in a manner analogous to Equation (3), Equation (7) augments physical diffusion by an amount proportional to $\Delta x$, and this numerical dissipation mitigates wiggles at the expense of smearing.

Shapiro and Pinder (1981) introduce a related upwinding technique for use with finite-element collocation. Their approach entails the use of Hermite cubic interpolating bases (see Prenter, 1975, Chapter 3), except they perturb the trial function in the convective term by a piecewise quartic biased in the upstream direction. Shapiro and Pinder present a detailed Fourier analysis showing the dissipative effects of their upstream weighting on the propagation of sharp fronts. 
There is another upwinding technique for finite-element collocation. Consider the standard implementation, in which the trial function his the form $\mathrm{N}$

$$
\hat{u}(x, t)=u_{\partial}(x)+\sum_{i=1}\left[u_{i}(t) H_{0 i}(x)+u_{i}{ }^{\prime}(t) H_{1 i}(x)\right]
$$

Here the coefficients $u_{i}(t), u_{i}{ }^{\prime}(t)$ approximate the nodal values $u\left(x_{j}, t\right)$, $\partial u\left(x_{i}, t\right) / \partial x$, respectively, and $\left\{H_{0 j}, H_{1 i}\right\}_{i=0}^{N+1}$ is the basis for Hermite cubic interpolation on $\Delta_{N}$. The standard collocation method, which has truncation error $\mathrm{O}\left(\Delta \mathbf{x}^{4}\right)$, requires the residual to vanish,

$$
\frac{\partial \hat{u}}{\partial t}\left(\bar{x}_{k}, t\right)+\frac{\partial \hat{u}}{\partial x}\left(\bar{x}_{k}, t\right)-P e^{-1} \frac{\partial^{2} \hat{u}}{\partial x^{2}}\left(\bar{x}_{k}, t\right)=0
$$

at each of $2 N$ collocation points $\bar{x}_{k}=x_{i}+\Delta x / 2 \pm \Delta x / \sqrt{3}, i=1, \ldots, N$. As Figure 1 shows, unless $\Delta x$ is sufficiently small the scheme generates wiggles near sharp fronts (Jensen and Finlayson, 1980).

A technique called upstream collocation (Allen and Pinder, 1983) offers a simple remedy to the wiggles, at the usual cost of smearing as shown in Figure 2. To implement the technique, simply shift the collocation points $\bar{x}_{k}$ in the convective term of Equation (8) to upstream points $\bar{x}_{k}^{*}=\bar{x}_{k}-\zeta \Delta x$. This gives

$$
\frac{\partial \hat{u}}{\partial t}\left(\bar{x}_{k}, t\right)+\frac{\partial \hat{u}}{\partial x}\left(\bar{x}_{k}^{*}, t\right)-P e^{-1} \frac{\partial^{2} \hat{u}}{\partial x^{2}}\left(\bar{x}_{k}, t\right)=0
$$

in which the differentiated basis-function values $H_{m i}{ }^{\prime}\left(\bar{x}_{k}{ }^{*}\right)$ appear in the convective term instead of $H_{m i}{ }^{\prime}\left(\bar{x}_{k}\right)$. By Taylor's theorem, the difference between these two values is $-\zeta \Delta x H_{m i}{ }^{\prime \prime}\left(\bar{x}_{k}\right)+\left(\zeta^{2} \Delta x^{2} / 2\right) H_{m i}{ }^{\prime \prime \prime}\left(\bar{x}_{k}\right)$. Thus, to within $O\left(\Delta x^{2}\right)$, Equation 9 is equivalent to

$$
\frac{\partial \hat{u}}{\partial t}\left(\bar{x}_{k}, t\right)+\frac{\partial \hat{u}}{\partial x}\left(\bar{x}_{k}, t\right)-\left(P e^{-1}+\zeta \Delta x\right) \frac{\partial^{2} \hat{u}}{\partial x^{2}}\left(\bar{x}_{k}, t\right)=0
$$

It is clear from this equation that numerical diffusion is again the mechanism by which the scheme mitigates wiggles.

Upstream collocation is closely related to an upwind Calerkin scheme introduced by Hughes (1978). This latter scheme involves numerical evaluation of the Galerkin integrals using quadrature points shifted upstream from the Gauss points. In fact, one can show an algebraic correspondence between upstream collocation and a variant of Hughes' method using reduced integration on Hermite trial spaces (Allen, to appear).

\section{A NONLINEAR HYPERBOLIC EQUATION}

Numerical dissipation makes upwinding attractive to modelers wishing to avoid wiggles in convection-dominated parabolic flows. It is precisely such motives that provoke justified ire in Gresho and Lee. There is, nevertheless, another motive for using upwind-biased schemes. Many physical systems combine minute dissipation with nonlinearity, obeying governing equations that are effectively hyperbolic. For these systems high-order discrete schemes may be mathematically inappropriate, not because they generate wiggles, but because 
they fail to converge. Upwinding techniques then provide reasonable alternatives.

The Buckley-Leverett problem furnishes a simple example of a nonlinear hyperbolic equation for which high-order approximations fail. Consider a typical Cauchy problem for this equation:

$$
\begin{gathered}
\frac{\partial S}{\partial t}+\frac{\partial}{\partial x} f(S)=0, \quad x, t>0 \\
S(0, t)=1-S_{o r}, S(x, 0)=S_{w r}
\end{gathered}
$$

Here $S$ stands for water saturation; $f(S)$ is a nonconvex, monotonically increasing function giving the flux of $S_{;}$and $S_{o r}$ and $S_{W r}$ are the minimum oil and water saturations, respectively, for the rock-fluid mixture. Equation (11) models immiscible flows in porous media in which capillarity exerts a negligible influence on fluid velocities. A prime feature of Equation (11) is the propagation of a saturation shock through the porous medium. This problem serves as a prototype for many kinds of nonlinear, hyperbolic or nearly hyperbolic systems of flow equations that occur in applications where convective forces are dominant.

It is widely known that spatially centered approximations to Equation (11) can converge to incorrect solutions. Allen and Pinder (1983), for example, examine the finite-element collocation approximation to this problem using both the conventional formulation and the upstream collocation scheme discussed above. As Figures 3 and 4 show, the conventional method predicts a saturation shock that is too slow and too strong, whereas the upstream method gives good approximations to the true shock. No amount of grid refinement can correct the failure of the conventional scheme. The difficulty here is not one of spurious wiggles; it is a deeper problem concerning the suitability of numerical methods from a mathematical standpoint.

Others have reported results similar to those displayed in Figures 3 and 4. Huyakorn and Pinder (1978) use the upstream-weighted Galerkin scheme reviewed above to overcome convergence difficulties with the standard Galerkin method in solving Equation (11). Mercer and Faust (1977) accomplish the same end by adding an adjustable capillary term to the discrete equations. Shapiro and Pinder (1980) use their upstream-weighted collocation method to produce convergent solutions to Equation (11). Indeed, upstream weighting has become standard practice for immiscible flow modeling in the oil industry (Aziz and Settari, 1979, Chapter 5 ).

\section{UNIQUENESS AND HYPERBOLIC CONSERVATION LAWS}

To see why upwind schemes converge for the Buckley-Leverett equation and similar problems, it is useful to review some mathematical facts about Equation (11). This equation is a quasilinear hyperbolic conservation law. When such equations have nonconvex flux functions like $f(S)$, one cannot expect Cauchy problems for the equations to possess classical solutions. Instead, one may have to settle for weak solutions, defined for Equation (11) by the integral criterion 


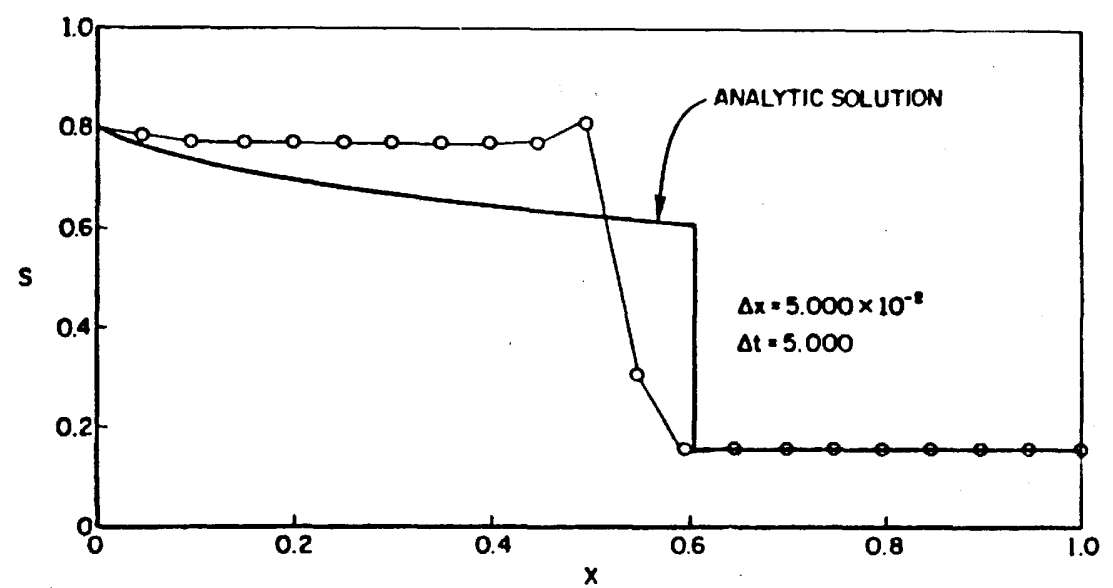

Figure 3. Solution to the Buckley-Leverett prol lem using standard collocation on finite elements.

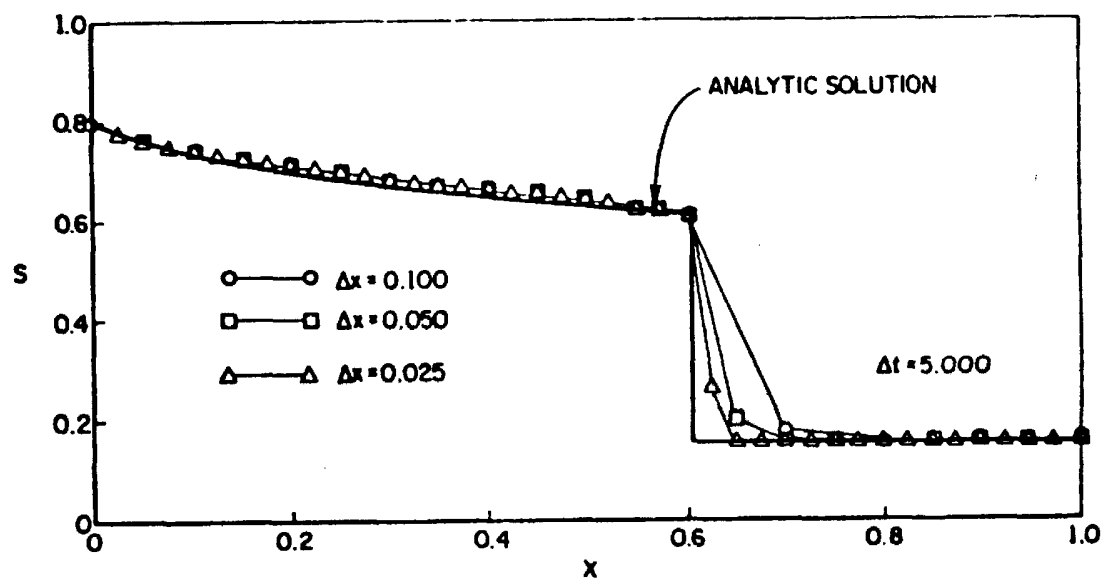

Figure 4. Solutions to the Buckley-Leverett problem using upstream collocation with spatial grids of varying mesh. 


$$
\int_{0}^{\infty} \int_{0}^{\infty}\left[S \frac{\partial \phi}{\partial t}+f(S) \frac{\partial \phi}{\partial x}\right] d x d t=0
$$

for any $c^{\infty}$ real-valued function $\phi(x, t)$ with compact support. This criterion reduces to Equation (11) when $S(x, t)$ is continuously differentiable but also admits solutions $S(x, t)$ having shocks.

However, Cauchy problems like Equation (11) may not have unique weak solutions. To guarantee uniqueness for general initial data requires an additional constraint. The correct constraint, or shock condition, requires the weak solution to depend continuously and stably on the initial data. Equivalently, characteristic curves emanating from both sides of a discontinuity must intersect the curve on which the initial data are given. Oleinik (1963) proves a uniqueness condition on weak solutions that is mathematically equivalent to the shock condition but has more immediate implications for discrete approximations. Her criterion essentially states that the solution to the hyperbolic equation must be the limit of solutions, for comparable data, to a parabolic equation differing from the hyperbolic one by a dissipative second-order term of vanishing influence. In gas dynamics, this second-order term is called "vanishing viscosity"; for the Buckley-Leverett problem the term "vanishing capillarity" is perhaps more appropriate.

High-order, spatially centered discretizations of the Buckley-Leverett problem, though formally consistent with Equation (11), yield approximate weak solutions that are physically and mathematically incorrect. From a physical standpoint the neglected capillary term in Equation (11), which has the form

$$
-\frac{\partial}{\partial x}\left[F(S) \frac{\partial S}{\partial x}\right], F(S) \geq 0
$$

exerts an important influence in a microscopic region of what appears to macroscopic observers as a saturation shock. Thus while the global effects of capillarity may be legitimately neglected in the macroscopic flow equation, some device must remain to guarantee that the solution $S(x, t)$ respect the microscopic physics. Artificial capillarity is such a device.

It is a relatively simple matter to see how various upwinded approximations contribute artificial capillarity. For example, an upstream-weighted difference approximation to Equation (11) that is analogous to Equation (3) yields a flux term that has the form

$$
\begin{gathered}
\Delta x^{-1}\left(f_{i}-f_{i-1}\right)=\left.\frac{\partial f}{\partial x}\right|_{i}-\left.(\Delta x / 2) \frac{\partial^{2} f}{\partial x^{2}}\right|_{i}+O\left(\Delta x^{2}\right) \\
=\left.\frac{\partial f}{\partial x}\right|_{i}-\left.(\Delta x / 2) \frac{\partial}{\partial x}\left[f^{\prime}(S) \frac{\partial S}{\partial x}\right]\right|_{i}+O\left(\Delta x^{2}\right)
\end{gathered}
$$

Since $f^{\prime}(S) \geq 0$, it is clear that the lowest error term here mimics the missing physical capillarity of Equation (12), while preserving consistency.

Similarly, the use of upstream-biased test functions in the Galerkin scheme analogous to Equation (7) yields approximations to the flux term of Equation 
(11) having an error $\mathrm{N}$

$$
\begin{aligned}
& \left\langle\partial \hat{f} / \partial x, \wedge_{j}\right\rangle=\sum_{i=1}^{N} f_{i} \alpha\left\langle L_{i}, A_{j}\right\rangle \\
& =-(\alpha / 2)\left[f_{j+1}-2 f_{j}+f_{j-1}\right]=-\left(\alpha \Delta x^{2} / 2\right)\left[\frac{\partial^{2} \hat{f}}{\partial x^{2}} \mid j+O\left(\Delta x^{2}\right)\right] \\
& =-\left(\alpha \Delta x^{2} / 2\right) \frac{\partial}{\partial x}\left[f^{\prime}(\hat{S}) \frac{\partial \hat{S}}{\partial x}\right] j+O\left(\Delta x^{2}\right)
\end{aligned}
$$

where $f_{i}=f\left(\hat{S}_{i}(t)\right)$ and $\hat{S}$ is the trial function approximating $S$. Notice that, parallelline Equation (13), the use of asymmetric test functions adds a numerical capillarity that is $O(\Delta x)$ smaller than the approximations to physical terms in Equation (11).

Upstrean collocation also adds artificial capillarity to the BuckleyLeverett problem. In this case we project the flux term of Equation (11) to a Hermite interpolating space and collocate the result $\partial \hat{f} / \partial x$ at poins $\bar{x}_{k}{ }^{*}=$ $\bar{x}_{k}-\zeta \Delta x$ upstream of the usual collocation points. A Taybor exparsion shows

$$
\begin{gathered}
\frac{\partial \hat{f}}{\partial x}\left(\bar{x}_{k}^{*}, t\right)-\frac{\partial \hat{f}}{\partial x}\left(\bar{x}_{k}, t\right)=-\zeta \Delta x \frac{\partial 2 \hat{f}}{\partial x^{2}}\left(\bar{x}_{k}, t\right)+O\left(\Lambda x^{2}\right. \\
=-\zeta \Delta x \frac{\partial}{\partial x}\left[f^{\prime}\left(\hat{S}\left(\bar{x}_{k}, t\right)\right) \frac{\partial \hat{S}}{\partial x}\left(\bar{x}_{k}, t\right)\right]+O\left(\Delta x^{2}\right)
\end{gathered}
$$

Again, the upwind scheme adds the necessary artificial dissipation in the form of a "vanishing capillarity."

Various physics give rise to effectively hyperbolic systems for which uniqueness of weak solutions is an important issue. For such systems formal neglect of dissipation, while arguably valid from an engineering viewpoint, necessitates a device like upwinding to guarantee qualitatively correct numerical solutions. Other examples of interest in water resources engineering include hydraulic jumps (Whitham, 1974, Chapter 13) and wetting fronts in variably saturated soils (Nakano, 1980).

\section{CONCLUSIONS}

Upwinding can serve two purposes: it can suppress wiggles or, for certain equations, it can guarantee convergence. As Gresho and Lee observe, the first purpose is largely cosmetic, and the attendant smearing may be a more difficult flaw to recognize than spurious oscillations. However, in the case of conservation laws with discontinuous weak solutions upwinding can be a legitimate practice. The aim in this second case is to formulate consistent approximations that have built-in mechanisms for accommodating the peculiarities of hyperbolic or nearly hyperbolic flows. The lower spatial accuracy inherent in upwind schemes is far preferable to the convergence failures of higher-order schemes. Indeed, in the neighborhood of a discontinuity the very notion of "order of accuracy ${ }^{*}$ can be problematic.

Despite the mathematical validity of upwinding, the problem of smearing remains. While numerical dissipation vanishes with $\Delta x$, in practice $\Delta x$ never vanishes and may be so large that artificial smearing is unacceptable. One of the most promising remedies to this difficulty is adaptive local grid refinement. Here one uses a spatial grid having smaller elements in portions of the flow 
field where steep solution gradients drive numerical dissipation. Algorithms combining local grid refinement with upwinding allow both for convergence to correct weak solutions and for the reduction of artificial smearing near sharp fronts. There is no denying the formidable coding difficulties in adaptive local grid refinement; however, progress in this field is encouraging (see Ewing, to appear).

\section{ACKNOWLEDGEMENTS}

Partial support for this work came from National Science Foundation grant number NSF-CEE-8111240 and from the Wyoming Water Research Center.

\section{REFERENCES}

1. Allen, M.B. (to appear), "How upstream collocation works," Int. J. Num. Meth. Engrg.

2. Allen, M.B., and Pinder, G.F. (1983), "Collocation simulation of multiphase porous-medium flow,' Soc. Pet. Eng. J. (February), 135-142.

3. Aziz, K., and Settari, A. (1979), Petroleum Reservoir Simulation, London: Applied Science.

4. Ewing, R. (to appear), 'Adaptive mesh refinement in reservoir simulation applications," to be presented at the International Conference on Accuracy Estimates and Adaptive Refinement in Finite Element Computations, Lisbon, Portugal, June, 1984.

5. Gresho, P.M., and Lee, R.L. (1980), 'Don't suppress the wiggles--they're telling you something $!^{*}$, in Finite Element Methods for Convection Dominated Flows, ed. by T.J.R. Hughes, New York: American Society of Mechanical Engineers, 37-61.

6. Heinrich, J.C., Huyakorn, P.S., Zienkiewicz, O.C., and Mitchell, A.R. (1977), 'An 'upwind' finite element scheme for two-dimensional convective transport equation," Int. J. Num. Meth. Engrg. 11, 131-143.

7. Hughes, T.J.R. (1978), "A simple scheme for developing upwind finite elements," Int. J. Num. Meth. Engrg. 12, 1359-1365.

8. Huyakorn, P.S., and Pinder, G.F. (1978), "A new finite element technique for the solution of two-phase flow through porous media," Adv. Water Resources 1:5, 285-298.

9. Jensen, O.K., and Finlayson, B.A. (1980), "Oscillation limits for weighted residual methods applied to convective diffusion equations," Int. J. Num. Meth. Engrg. 15, 1681-1689.

10. Mercer, J.W., and Faust, C.R. (1977), "The application of finite-element techniques to immiscible flow in porous media," presented at the First International Conference on Finite Elements in Water Resources, Princeton, NJ, July, 1976.

11. Nakano, Y. (1980), "Application of recent results in functional analysis to the problem of wetting fronts," Water Res. Research 16, 314-318.

12. Oleinik, O.A. (1963), "Construction of a generalized solution of the Cauchy problem for a quasi-linear equation of the first order by the introduction of a 'vanishing viscosity'," Am. Math. Soc. Transl. (Ser. 2), 33, 277-283.

13. Prenter, P.M. (1975), Splines and Variational Methods, New York: Wiley.

14. Shapiro, A.M., and Pinder, G.F. (1981), "Analysis of an upstream weighted approximation to the transport equation," 1. Comp. Phys. 39 
46-71.

15. Shapiro, A.M., and Pinder, G.F. 1982), "Solution of immiscible displacement in porous media using the collocation finite element method," presented at the Fourth International Conference on Finite Elements in Water Resources, Hannover, FRC, June, 1982.

16. Von Neumann, J., and Richtmyer, R.D. (1950), "A method for the numerical calculations of hydrodynamical shocks, J. Appl. Phys. 21, 232-237.

17. Whitman, G.B. (1974), Linear and Nonlinear Waves, New York: Wiley. 